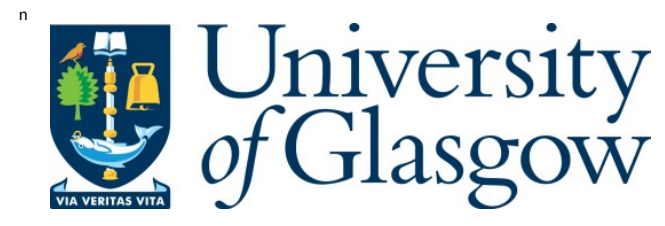

Y ang , D., Y e, Z., N obre, A. M ., Du, H., Walsh, W. M ., Lim, L. I., and Reindl, T. (2014) Bidirectional irradiance transposition based on the Perez model. Solar Energy, 110 . pp. 768-780. ISSN 0038-092X

Copyright @ 2014 Elsevier Ltd.

A copy can be downloaded for personal non-commercial research or study, without prior permission or charge

Content must not be changed in any way or reproduced in any format or medium without the formal permission of the copyright holder(s)

http://eprints.gla.ac.uk/100843/

Deposited on: 15 J anuary 2015

Enlighten - Research publications by members of the University of Glasgow http://eprints.gla.ac.uk 


\title{
Bidirectional irradiance transposition based on the Perez model
}

\author{
Dazhi Yang,b,*, Zhen Yec, André M. Nobre ${ }^{\mathrm{a}}$, Hui Du ${ }^{\mathrm{a}}$, Wilfred M. Walsh ${ }^{\mathrm{a}}$, \\ Lihong Idris Lim ${ }^{\mathrm{d}}$, Thomas Reindl ${ }^{\mathrm{a}}$ \\ ${ }^{a}$ Solar Energy Research Institute of Singapore (SERIS), National University of \\ Singapore, 7 Engineering Drive 1, Block E3A, Singapore 117574, Singapore \\ ${ }^{b}$ Department of Electrical and Computer Engineering, National University of Singapore, \\ 4 Engineering Drive 3, Block E4, Singapore 117583, Singapore \\ ${ }^{c}$ Module Technology, REC Cells Pte Ltd., 20 Tuas South Avenue 14, Singapore 637312, \\ Singapore \\ ${ }^{d}$ Department of Electronic Systems, University of Glasgow (Singapore), 535 Clementi \\ Road, Singapore 599489, Singapore
}

\begin{abstract}
The Perez irradiance model offers a practical representation of solar irradiance by considering the sky hemisphere as a three part geometrical framework, namely, circumsolar disc, horizon band and the isotropic background. Furthermore, the simplified Perez diffuse irradiance model, commonly known as the Perez transposition model, is one of the most widely adopted models in tilted irradiance assessments. Although the set of model coefficients reported by Perez et al. (1990) is often considered to be at an asymptotic level of optimization, later analyses have shown that coefficients which are adjusted to local conditions perform better than the original set.

The model coefficients can be adjusted locally based on multiple datasets of diffuse and global irradiance on tilted and horizontal planes. In this paper, we present a different approach to adjust the coefficients, by using only measurements of global irradiance on tilted and horizontal planes from a tropical site, Singapore. A complete set of mathematical solutions to the inverse problem, i.e., converting irradiance from tilt to horizontal is also proposed. The data can then be used to generate irradiance maps from in-plane irradiance measurements at photovoltaic (PV) systems. Such maps are get-
\end{abstract}

\footnotetext{
*Corresponding author. Tel.: +65 91590888.

Email address: seryangd@nus.edu.sg, yangdazhi.nus@gmail.com (Dazhi Yang)
} 
ting more relevant for PV grid integration due to the variable nature of solar power output.

Keywords: Perez model, reference cells, tropical regions, inverse transposition

\section{List of symbols.}

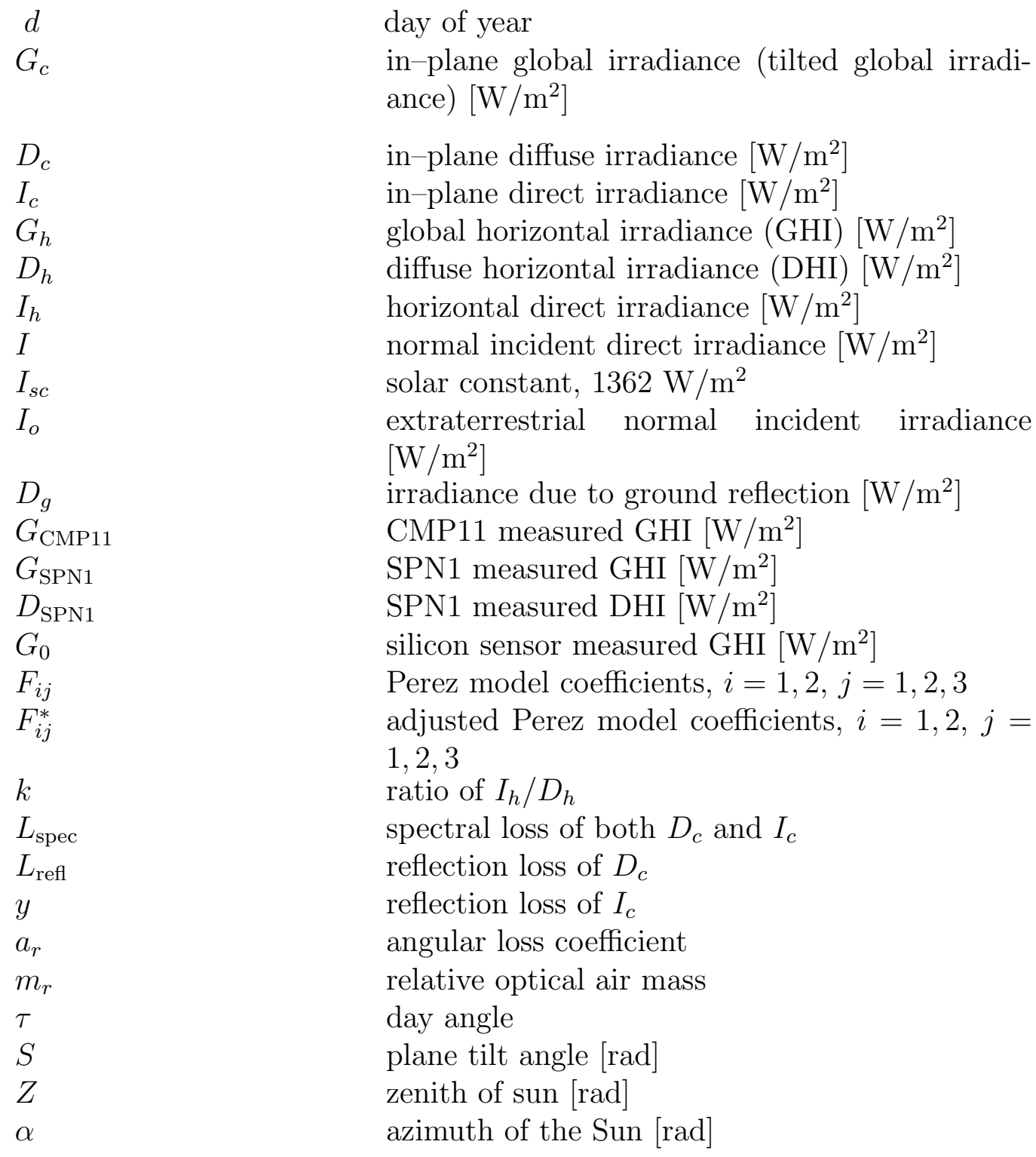




$\begin{array}{ll}\beta & \text { azimuth of PV }[\mathrm{rad}] \\ \delta & \text { foreground's albedo } \\ \Delta & \text { sky's brightness } \\ \varepsilon & \text { sky's clearness } \\ \theta & \text { solar incidence angle on plane of array (POA) } \\ \vartheta & \text { [rad }] \\ & \text { solar incidence angle on POA }\left[^{\circ}\right]\end{array}$

\section{Introduction}

Conversion of solar irradiance measurements from the horizontal to a tilted surface (or vice versa) is a problem commonly encountered in the design of solar energy systems. Many models address this problem, with early results tracing back to (Liu and Jordan, 1962) which assume an isotropic sky hemisphere. However the anisotropic nature of diffuse irradiance has been the well-known source of error associated with this assumption (Ma and Iqbal, 1983). To account for that, various anisotropic models have been proposed (Skartveit and Olseth, 1986; Hay, 1979; Klucher, 1979; Temps and Coulson, 1977). Various versions of the Perez model (Perez et al., 1990, 1988, 1987,1986 ) are the most universal (for all locations) and most frequently used transposition models (Myers, 2012; Matagne and Bachtiri, 2014). Many studies have applied the Perez model for various applications, e.g., Khoo et al. (2014) used the Perez model to maximize PV array outputs by adjusting its orientation and tilt; Zomer et al. (2013) used the Perez model while analyzing building-integrated photovoltaic systems.

The Perez model defines three main irradiance regimes (the background hemisphere, the circumsolar disk and a band near the horizon) whose instantaneous characteristics are determined by atmospheric phenomena such as cloudiness and turbidity. Ever since its introduction, the authors of the Perez model have emphasized the need to tailor the model's coefficients to the locale at which it is applied. We seek to explore the Perez model's applicability to tropical skies, which have rarely been benchmarked against irradiance models ${ }^{1}$, but which will become increasingly important as developing nations in the global equatorial zone implement solar technologies. To this end, we

\footnotetext{
${ }^{1}$ No tropical location is used in the canonical 1990 formulation of the model (Perez et al., 1990).
} 
fit local model coefficients to the Perez transposition model in section 2. We also consider the Perez model from a mathematical point of view in this paper: we find the solutions to the inverse transposition problem (irradiance conversion from tilted surfaces to horizontal) in section 3.

The simplified Perez diffuse irradiance model (Perez et al., 1987) is given by

$$
D_{c}=D_{h}\left[\left(1-F_{1}\right) \frac{1+\cos S}{2}+F_{1} \frac{a}{b}+F_{2} \sin S\right],
$$

where $D_{h}$ and $D_{c}$ are diffuse irradiance on horizontal plane and tilted plane, respectively; $S$ is the plane tilt angle;

$$
\begin{aligned}
a & =\max \{0, \cos \theta\}, \\
b & =\max \left\{\cos 85^{\circ}, \cos Z\right\},
\end{aligned}
$$

given by Perez et al. (1990) are a result of the approximation by assuming that all circumsolar energy originates from a point source; $\theta$ is the solar incidence angle; $Z$ is the zenith angle;

$$
\begin{aligned}
& F_{1}=\max \left\{0, F_{11}(\varepsilon)+\Delta F_{12}(\varepsilon)+Z F_{13}(\varepsilon)\right\}, \\
& F_{2}=F_{21}(\varepsilon)+\Delta F_{22}(\varepsilon)+Z F_{23}(\varepsilon),
\end{aligned}
$$

are defined as reduced brightness coefficients where $\Delta=D_{h} \times m_{r} / I_{o}$ is the sky's brightness. $m_{r} \approx 1 / \cos Z$ is the relative optical air mass; $I_{o} \approx I_{s c}(1+$ $0.033 \cos \tau$ ), where $I_{s c}=1362 \mathrm{~W} / \mathrm{m}^{2}$ is the solar constant, $\tau=2 \pi d / 365, d$ is the day number of a year and ranges from 0 on 1 January to 364 on 31 December;

$$
\varepsilon=\frac{1+I / D_{h}+\kappa Z^{3}}{1+\kappa Z^{3}}
$$

is the sky's clearness, where $I=I_{h} / \cos Z$ is the direct normal irradiance, and the constant $\kappa=1.041$ when $Z$ is in radians. The unknown parameters $F_{11}, F_{12}, F_{13}, F_{21}, F_{22}, F_{23}$ can be determined empirically from measurements of the irradiance on tilted surfaces. The set of $F_{11}, F_{12}, F_{13}, F_{21}$, $F_{22}, F_{23}$ has evolved a few times. The most widely accepted set of coefficients is given by Perez et al. (1990) as shown in Table 1, which is based on data from Albany (USA), Geneva (Switzerland), Los Angeles (USA), Albuquerque (USA), Phoenix (USA), Cape Canaveral (USA), Osage (USA), Trappes (France), and Carpentras (France). 
Table 1: Perez model coefficients for irradiance as a function of the sky's clearness index $\varepsilon$ (cf. Myers, 2013).

\begin{tabular}{ccccccc}
\hline$\varepsilon$ & $F_{11}$ & $F_{12}$ & $F_{13}$ & $F_{21}$ & $F_{22}$ & $F_{23}$ \\
\hline$[1,1.065)$ & -0.0083 & 0.5877 & -0.0621 & -0.0596 & 0.0721 & -0.0220 \\
{$[1.065,1.23)$} & 0.1299 & 0.6826 & -0.1514 & -0.0189 & 0.0660 & -0.0289 \\
{$[1.23,1.5)$} & 0.3297 & 0.4869 & -0.2211 & 0.0554 & -0.064 & -0.0261 \\
{$[1.5,1.95)$} & 0.5682 & 0.1875 & -0.2951 & 0.1089 & -0.1519 & -0.0140 \\
{$[1.95,2.8)$} & 0.8730 & -0.3920 & -0.3616 & 0.2256 & -0.4620 & 0.0012 \\
{$[2.8,4.5)$} & 1.1326 & -1.2367 & -0.4118 & 0.2878 & -0.823 & 0.0559 \\
{$[4.5,6.2)$} & 1.0624 & -1.5999 & -0.3589 & 0.2642 & -1.1272 & 0.1311 \\
{$[6.2,+\infty)$} & 0.6777 & -0.3273 & -0.2504 & 0.1516 & -1.3765 & 0.2506 \\
\hline
\end{tabular}

Note: The notation $[a, b)$ is used to indicate an interval from $a$ to $b$ that is inclusive of $a$ but exclusive of $b$.

Although Table 1 is considered to be at an asymptotic level of optimization, the locations used to fit the original set of coefficients do not include a tropical site. Meteorological differences between the original sites and tropical regions may therefore affect the performance of the transposition model. Singapore is located near the equator with a latitude of $1.3^{\circ} \mathrm{N}$ and has a tropical climate. It is therefore well-suited for investigating the effect of locally (under a tropical weather condition) adjusted model coefficients. We explicitly express the mathematical derivations here to facilitate further applications in other locations where model coefficients adjustment may apply.

\section{Model coefficients adjustment}

We use a least square approach to adjust the model coefficients $F_{11}, F_{12}$, $F_{13}, F_{21}, F_{22}, F_{23}$. Substituting Eqns. (4) and (5) into Eqn. (1) yields

$$
\begin{aligned}
D_{c}= & D_{h} \frac{1+\cos S}{2}+D_{h}\left(\frac{a}{b}-\frac{1+\cos S}{2}\right)\left(F_{11}+\Delta F_{12}+Z F_{13}\right) \\
& +D_{h} \sin S\left(F_{21}+\Delta F_{22}+Z F_{23}\right) .
\end{aligned}
$$

Given $n$ tilted sensors and $m$ samples of $D_{h}$ (and $D_{c}$ correspondingly), the equivalent linear algebra of Eqn. (7) is

$$
\mathrm{AX}=\mathrm{Y},
$$

where

$$
\mathbf{X}=\left(\begin{array}{llllll}
F_{11} & F_{12} & F_{13} & F_{21} & F_{22} & F_{23}
\end{array}\right)^{\prime}, \quad \in \mathbb{R}^{6}
$$




$$
\mathbf{A}=\left(\begin{array}{cccccc}
A_{1}^{1,1} & A_{2}^{1,1} & A_{3}^{1,1} & A_{4}^{1,1} & A_{5}^{1,1} & A_{6}^{1,1} \\
\vdots & \vdots & \vdots & \vdots & \vdots & \vdots \\
A_{1}^{1, m} & A_{2}^{1, m} & A_{3}^{1, m} & A_{4}^{1, m} & A_{5}^{1, m} & A_{6}^{1, m} \\
\vdots & \vdots & \vdots & \vdots & \vdots & \vdots \\
A_{1}^{n, 1} & A_{2}^{n, 1} & A_{3}^{n, 1} & A_{4}^{n, 1} & A_{5}^{n, 1} & A_{6}^{n, 1} \\
\vdots & \vdots & \vdots & \vdots & \vdots & \vdots \\
A_{1}^{n, m} & A_{2}^{n, m} & A_{3}^{n, m} & A_{4}^{n, m} & A_{5}^{n, m} & A_{6}^{n, m}
\end{array}\right), \quad \in \mathbb{R}^{n m \times 6}
$$

with $A_{1}^{i, j}=D_{h}^{j}\left[a_{i, j} / b_{j}-\left(1+\cos S_{i}\right) / 2\right], A_{2}^{i, j}=\Delta_{j} A_{1}^{i, j}, A_{3}^{i, j}=Z_{j} A_{1}^{i, j}, A_{4}^{i, j}=$ $D_{h}^{j} \sin S_{i}, A_{5}^{i, j}=\Delta_{j} A_{4}^{i, j}, A_{6}^{i, j}=Z_{j} A_{4}^{i, j}, i=1, \cdots, n, j=1, \cdots, m$.

$$
\mathbf{Y}=\left(\begin{array}{lllllll}
Y_{1,1} & \cdots & Y_{1, m} & \cdots & Y_{n, 1} & \cdots & Y_{n, m}
\end{array}\right)^{\prime}, \quad \in \mathbb{R}^{n m}
$$

with $Y_{i, j}=D_{c}^{i, j}-D_{h}^{j}\left(1+\cos S_{i}\right) / 2, i=1, \cdots, n, j=1, \cdots, m$. The least square solution to Eqn. (8) is given by

$$
\mathbf{X}=\left(\mathbf{A}^{\prime} \mathbf{A}\right)^{-1} \mathbf{A}^{\prime} \mathbf{Y}
$$

This least squares method is described by Perez et al. (1988). To establish an accurate fit, the method requires $n$ sets of measurements of tilted diffuse irradiance and a set of horizontal diffuse irradiance measurements (each set has $m$ samples), i.e., $D_{c}^{i, j}$ and $D_{h}^{j}$. However in practice, tilted diffuse irradiance measurements are scarce. This is particularly true in developing countries where long term, precisely calibrated irradiance datasets are often not available. Cost-effective reference cells can however be used to measure global tilted irradiance, $G_{c}=D_{c}+I_{c}$, where $I_{c}$ is the in-plane direct irradiance. Such sensors are commonly installed in PV systems for system monitoring and it is likely that such data becomes widely available as PV systems proliferate globally. We therefore propose a novel approach that circumvents the need for tilted diffuse component observations. We model $G_{c}$ and construct $D_{c}$ and $I_{c}$ mathematically.

Two types of devices are commonly used to perform radiometric measurements, namely, thermopile pyranometers and reference cells. Meydbray et al. (2012) pointed out three disadvantages of reference cells as compared to a tilted pyranometer: 1) reference cells suffer from spectral loss as they have a narrow wavelength response, 2) reference cells suffer from reflection losses owing to its flat surface design and 3) the linearity of the reference cells' output signals is affected by temperature, this however can be corrected with an 
on-board temperature sensor. Therefore we need to account for the spectral and reflection losses when we use reference cell measurements. We embed the losses in our formulations as follows.

\subsection{Derivation of $D_{c}$}

The irradiance due to ground reflection (Temps and Coulson, 1977), $D_{g}$, is given by

$$
D_{g}=\frac{1}{2} \delta\left(I_{h}+D_{h}\right)(1-\cos S)\left[1+\sin ^{2}(Z / 2)\right]|\cos (\alpha-\beta)|,
$$

must be added into $D_{c}$, where $\delta$ is the foreground's albedo, $I_{h}$ is the horizontal direct irradiance, $\alpha$ and $\beta$ are the azimuth of sun and plane respectively. We denote the reflection loss and spectral loss as $L_{\text {refl }}$ and $L_{\text {spec }}$, then Eqn. (7) becomes

$$
\begin{aligned}
D_{c}= & 0.5 \delta\left(I_{h}+D_{h}\right)(1-\cos S)\left[1+\sin ^{2}(Z / 2)\right]|\cos (\alpha-\beta)|\left(1-L_{\mathrm{reff}}\right)\left(1-L_{\mathrm{spec}}\right) \\
& +D_{h} \frac{1+\cos S}{2}\left(1-L_{\mathrm{refl}}\right)\left(1-L_{\mathrm{spec}}\right) \\
& +D_{h}\left(\frac{a}{b}-\frac{1+\cos S}{2}\right)\left(1-L_{\mathrm{reff}}\right)\left(1-L_{\mathrm{spec}}\right)\left(F_{11}+\Delta F_{12}+Z F_{13}\right) \\
& +D_{h} \sin S\left(1-L_{\mathrm{reff}}\right)\left(1-L_{\mathrm{spec}}\right)\left(F_{21}+\Delta F_{22}+Z F_{23}\right) .
\end{aligned}
$$

Having obtained the $D_{c}$ representation, to construct $G_{c}$, we express $I_{c}$ as follows.

\subsection{Derivation of $I_{c}$}

$I_{c}$ can be calculated from $I_{h}$ deterministically by geometry as:

$$
I_{c}=I_{h} \frac{\cos \theta}{\cos Z} .
$$

If considering spectral and reflection losses, Eqn. (15) becomes:

$$
I_{c}=I_{h} \frac{\cos \theta}{\cos Z}(1-y)\left(1-L_{\mathrm{spec}}\right) .
$$


Here we use a set of parameter $y$ provided by the Fraunhofer Institute for Solar Energy Systems (ISE),

$$
y= \begin{cases}0, & \text { if } \vartheta \in\left[0,30^{\circ}\right) ; \\ 0.0006\left(\vartheta-30^{\circ}\right), & \text { if } \vartheta \in\left[30^{\circ}, 40^{\circ}\right) ; \\ 0.006+0.0012\left(\vartheta-40^{\circ}\right), & \text { if } \vartheta \in\left[40^{\circ}, 50^{\circ}\right) ; \\ 0.018+0.0029\left(\vartheta-50^{\circ}\right), & \text { if } \vartheta \in\left[50^{\circ}, 60^{\circ}\right) ; \\ 0.047+0.0068\left(\vartheta-60^{\circ}\right), & \text { if } \vartheta \in\left[60^{\circ}, 65^{\circ}\right) ; \\ 0.081+0.0098\left(\vartheta-65^{\circ}\right), & \text { if } \vartheta \in\left[65^{\circ}, 70^{\circ}\right) ; \\ 0.13+0.0166\left(\vartheta-70^{\circ}\right), & \text { if } \vartheta \in\left[70^{\circ}, 75^{\circ}\right) ; \\ 0.213+0.0276\left(\vartheta-75^{\circ}\right), & \text { if } \vartheta \in\left[75^{\circ}, 80^{\circ}\right) ; \\ 0.351+0.047\left(\vartheta-80^{\circ}\right), & \text { if } \vartheta \in\left[80^{\circ}, 85^{\circ}\right) ; \\ 0.586+0.0828\left(\vartheta-85^{\circ}\right), & \text { if } \vartheta \in\left[85^{\circ}, 90^{\circ}\right),\end{cases}
$$

to account for the reflection loss of $I_{c}$ on the glass surface of silicon sensors with $\vartheta=\theta \times 180^{\circ} / \pi$. An alternative analytic representation of the reflection loss is given in Appendix A.

Substituting Eqns. (14) and (16) into the equation $G_{c}=I_{c}+D_{c}$, and dividing $D_{h}$ on both sides yields:

$$
\begin{aligned}
\frac{G_{c}}{D_{h}}= & \frac{I_{h} \cos \theta(1-y)\left(1-L_{\text {spec }}\right)}{D_{h} \cos Z} \\
& +0.5 \delta \frac{G_{h}}{D_{h}}(1-\cos S)\left[1+\sin ^{2}(Z / 2)\right]|\cos (\alpha-\beta)|\left(1-L_{\text {refl }}\right)\left(1-L_{\text {spec }}\right) \\
& +\frac{1+\cos S}{2}\left(1-L_{\text {refl }}\right)\left(1-L_{\text {spec }}\right) \\
& +\left(\frac{a}{b}-\frac{1+\cos S}{2}\right)\left(1-L_{\text {refl }}\right)\left(1-L_{\text {spec }}\right)\left(F_{11}+\Delta F_{12}+Z F_{13}\right) \\
& +\sin S\left(1-L_{\text {refl }}\right)\left(1-L_{\text {spec }}\right)\left(F_{21}+\Delta F_{22}+Z F_{23}\right) .
\end{aligned}
$$

where $G_{h}=I_{h}+D_{h}$ is the global horizontal irradiance. We then write Eqn. (18) into linear algebra form:

$$
\mathbf{A}^{*} \mathbf{X}=\mathbf{Y}^{*}
$$


where

$$
\mathbf{A}^{*}=\left(\begin{array}{cccccc}
H_{1}^{1,1} & \Delta_{1,1} H_{1}^{1,1} & Z_{1} H_{1}^{1,1} & H_{2}^{1,1} & \Delta_{1,1} H_{2}^{1,1} & Z_{1} H_{2}^{1,1} \\
\vdots & \vdots & \vdots & \vdots & \vdots & \vdots \\
H_{1}^{1, m} & \Delta_{1, m} H_{1}^{1, m} & Z_{m} H_{1}^{1, m} & H_{2}^{1, m} & \Delta_{1, m} H_{2}^{1, m} & Z_{m} H_{2}^{1, m} \\
\vdots & \vdots & \vdots & \vdots & \vdots & \vdots \\
H_{1}^{n, 1} & \Delta_{n, 1} H_{1}^{n, 1} & Z_{1} H_{1}^{n, 1} & H_{2}^{n, 1} & \Delta_{n, 1} H_{2}^{n, 1} & Z_{1} H_{2}^{n, 1} \\
\vdots & \vdots & \vdots & \vdots & \vdots & \vdots \\
H_{1}^{n, m} & \Delta_{n, m} H_{1}^{n, m} & Z_{m} H_{1}^{n, m} & H_{2}^{n, m} & \Delta_{n, m} H_{2}^{n, m} & Z_{m} H_{2}^{n, m}
\end{array}\right), \quad \in \mathbb{R}^{n m \times 6}
$$

with

$$
\begin{aligned}
& H_{1}^{i, j}=\left(\frac{a_{i, j}}{b_{j}}-\frac{1+\cos S_{i}}{2}\right)\left(1-L_{\mathrm{refl}}\right), \\
& H_{2}^{i, j}=\sin S_{i}\left(1-L_{\mathrm{refl}}\right)\left(1-L_{\mathrm{spec}}\right)
\end{aligned}
$$

and

$$
\mathbf{Y}^{*}=\left(\begin{array}{lllllll}
Y_{1,1}^{*} & \cdots & Y_{1, m}^{*} & \cdots & Y_{n, 1}^{*} & \cdots & Y_{n, m}^{*}
\end{array}\right)^{\prime}, \quad \in \mathbb{R}^{n m}
$$

with

$$
\begin{aligned}
Y_{i, j}^{*}= & \frac{G_{c}^{i, j}}{D_{h}^{j}}-\left(1-L_{\mathrm{reff}}\right)\left(1-L_{\mathrm{spec}}\right) \times \\
& \left(\frac{1+\cos S_{i}}{2}+0.5 \delta \frac{G_{h}^{j}}{D_{h}^{j}}\left(1-\cos S_{i}\right)\left[1+\sin ^{2}\left(Z_{j} / 2\right)\right]\left|\cos \left(\alpha_{j}-\beta\right)\right|\right) \\
& -\frac{I_{h}^{i, j} \cos \theta_{i, j}\left(1-y_{i, j}\right)\left(1-L_{\mathrm{spec}}\right)}{D_{h}^{j} \cos Z_{j}} .
\end{aligned}
$$

Then, the parameters of $F_{11}, F_{12}, F_{13}, F_{21}, F_{22}, F_{23}$ can be optimized by the least square method:

$$
\mathbf{X}=\left(\mathbf{A}^{* \prime} \mathbf{A}^{*}\right)^{-1} \mathbf{A}^{* \prime} \mathbf{Y}^{*}
$$

Both Eqns. (25) and (12) can be used to adjust the Perez model coefficients depending on available data. Practically, data are first binned into 8 categories following the classification of $\varepsilon$ in Table 1 . For each subset of data, 6 model coefficients of the respective bin are determined using the least square methods shown above. The procedure is repeated 8 times for a total of 48 coefficients. 


\subsection{Derivation of $\alpha, \theta$ and $Z$}

By inspecting each components of Eqn. (25), we need to calculate $\alpha, \theta$ and $Z$. They are three frequently encountered solar positioning parameters. There are many simplified approximations for these parameter (e.g. Michalsky, 1988; Masters, 2004). To ensure sufficient accuracy, we use the Solar Position Algorithm (Reda and Andreas, 2004, 2008) from National Renewable Energy Laboratory to calculate the azimuth of the sun, the incidence angle and the zenith angle. The details of this algorithm can be found in the open source C program.

\subsection{Performance}

All measurements used in this paper are taken from the meteorological station at the Solar Energy Research Institute of Singapore (SERIS). The horizontal global irradiance, $G_{h}$, is measured by three devices: a silicon sensor ( $\pm 5 \%$ uncertainty), a pyranometer (CMP11 from Kipp \& Zonen, $\pm 3 \%$ uncertainty) and a pyranometer (SPN1 from Delta-T, $\pm 5 \%$ uncertainty); horizontal diffuse irradiance is measured by the same pyranometer (SPN1); in-plane global irradiance is measured by four other silicon sensors with different tilts: $10^{\circ}, 20^{\circ}, 30^{\circ}$ and $40^{\circ}$, with the a same azimuth of $64^{\circ} \mathrm{NE}$. All silicon sensors are calibrated at the Fraunhofer ISE CalLab with on-board temperature sensors to perform temperature correction. Measurements are sampled with overall historical data availability higher than $99.7 \%$. The sampling rate is 1 second; and the data is logged as 1 min averages. Figure 1 shows a photograph of the irradiance measurement station located on the roof of SERIS.

The Perez model has been used mostly for hourly application despite its initial instantaneous design. To ensure sufficient samples, an hourly averaged data set over the entire year 2013, is used to adjust the Perez model coefficients; data from 2014 January to 2014 May are used for validation. Data points with $Z>87^{\circ}$ and $\vartheta>90^{\circ}$ are not considered. For horizontal irradiance inputs, we use interpolated values:

$$
\begin{aligned}
D_{h} & =G_{\mathrm{CMP} 11} \frac{D_{\mathrm{SPN} 1}}{G_{\mathrm{SPN} 1}} \\
I_{h} & =G_{\mathrm{CMP} 11}-D_{h}
\end{aligned}
$$

since CMP11 is an industrial standard sensor with higher accuracy than SPN1. The ratio of diffuse/global irradiance is derived from the SPN1 data. 


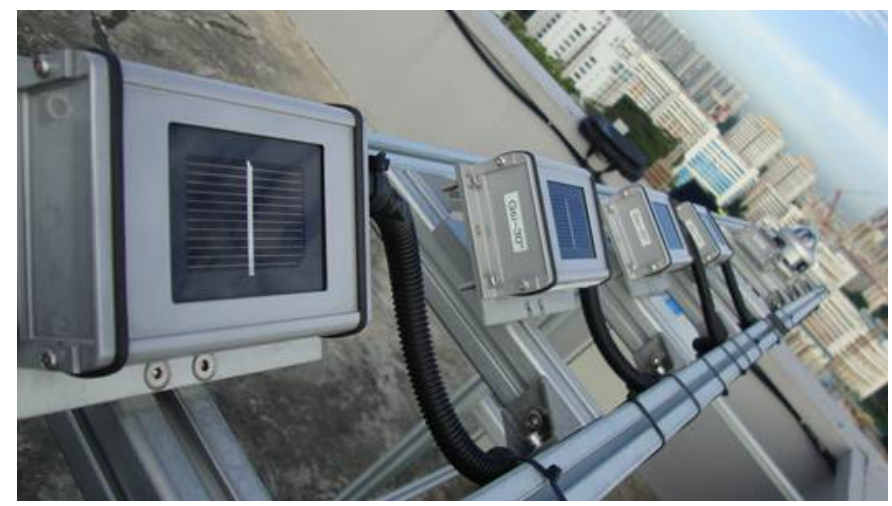

Figure 1: Photograph of the irradiance measurement station located on the rooftop of the Solar Energy Research Institute of Singapore (SERIS).

$G_{\mathrm{CMP} 11}, D_{\mathrm{SPN} 1}$ and $G_{\mathrm{SPN} 1}$ are readings from CMP11 (global irradiance) and SPN1 (diffuse and global irradiance), respectively.

Following procedures described in section 2.1 to 2.3, the adjusted set of $F_{11}^{*}, F_{12}^{*}, F_{13}^{*}, F_{21}^{*}, F_{22}^{*}, F_{23}^{*}$ are calculated, as shown in Table 2 . The readings from the 4 tilted silicon sensors (at $10^{\circ}, 20^{\circ}, 30^{\circ}$, and $40^{\circ}$ ) are used for fitting the new parameters. We use mean bias error $(\mathrm{MBE})$ in $\mathrm{W} / \mathrm{m}^{2}$, percentage root mean square error (RMSE) in \% and expanded uncertainty at $95 \%$ confidence interval $\left(U_{95}\right)$ in \% as our error metrics for the evaluation. The error calculations are shown below:

$$
\mathrm{MBE}=\frac{1}{m} \sum\left(\hat{G}_{c}-G_{c}\right),
$$

where $m$ is number of samples and $\hat{G}_{c}$ is the modeled value.

$$
\mathrm{RMSE}=\frac{\sqrt{\frac{1}{m} \sum\left(\hat{G}_{c}-G_{c}\right)^{2}}}{\frac{1}{m} \sum G_{c}},
$$

and

$$
U_{95}=1.96 \times \frac{\sqrt{\frac{1}{m} \sum\left(\hat{G}_{c}-\mathrm{MBE}-G_{c}\right)^{2}}}{\frac{1}{m} \sum G_{c}},
$$


where 1.96 is the coverage factor for a $95 \%$ confidence level. Table 3 shows the MBE, RMSE and $U_{95}$ of irradiance conversion results to various tilts using standard Perez model with coefficient sets $F$ and $F^{*}$.

Table 2: Adjusted Perez model coefficients for irradiance using irradiance data from Singapore

\begin{tabular}{ccccccc}
\hline$\varepsilon$ & $F_{11}^{*}$ & $F_{12}^{*}$ & $F_{13}^{*}$ & $F_{21}^{*}$ & $F_{22}^{*}$ & $F_{23}^{*}$ \\
\hline$[1,1.065)$ & 0.0485 & 0.5018 & -0.1070 & 0.1976 & -0.4549 & 0.0051 \\
{$[1.065,1.23)$} & 0.3239 & 0.3845 & -0.2828 & 0.2682 & -0.5768 & -0.0013 \\
{$[1.23,1.5)$} & 0.5479 & 0.3355 & -0.4283 & 0.1301 & -0.1287 & 0.0166 \\
{$[1.5,1.95)$} & 0.6305 & 0.8401 & -0.5762 & 0.1082 & -0.1478 & 0.1072 \\
{$[1.95,2.8)$} & 1.1469 & -0.1501 & -0.7686 & 0.0979 & -0.5319 & 0.1802 \\
{$[2.8,4.5)$} & 1.9053 & -2.7319 & -0.9979 & 0.0982 & -0.3522 & 0.2872 \\
{$[4.5,6.2)$} & 1.0591 & 0.4510 & -0.5650 & 0.1126 & -0.5392 & 0.2710 \\
{$[6.2,+\infty)$} & 0.0949 & -9.0339 & -0.4739 & 0.0742 & -0.2914 & 0.3272 \\
\hline
\end{tabular}

Table 3: Error comparison for irradiance conversion from horizontal to tilted planes in Singapore using the Perez model with coefficient sets $F$ (original) and $F^{*}$ (adjusted). $\overline{G_{c}}$ is the mean observed tilted irradiance in $\mathrm{W} / \mathrm{m}^{2}$.

\begin{tabular}{cccccccc}
\hline \multirow{2}{*}{$S$} & $\bar{G}_{c}\left[\mathrm{~W} / \mathrm{m}^{2}\right]$ & \multicolumn{2}{c}{$\mathrm{MBE}\left[\mathrm{W} / \mathrm{m}^{2}\right]$} & \multicolumn{2}{c}{$\mathrm{RMSE}[\%]$} & \multicolumn{2}{c}{$U_{95}[\%]$} \\
\cline { 3 - 6 } & & $F$ & $F^{*}$ & $F$ & $F^{*}$ & $F$ & $F^{*}$ \\
\hline $10^{\circ}$ & 439.5 & 2.54 & 4.21 & 2.31 & 2.26 & 4.08 & 4.01 \\
$20^{\circ}$ & 442.2 & -2.39 & 0.63 & 2.73 & 2.13 & 4.38 & 4.17 \\
$30^{\circ}$ & 426.5 & -0.01 & 4.02 & 3.57 & 3.19 & 6.24 & 5.96 \\
$40^{\circ}$ & 406.3 & -3.17 & 1.47 & 4.60 & 4.00 & 8.12 & 7.81 \\
\hline
\end{tabular}

We further describe the errors by showing the scatter plots of the modeled values versus the measurements (see Figure 2). Minimal deviations from the $45^{\circ}$ lines are observed in these plots showing unbiased (small MBE) estimations under both sets of model coefficients. We conclude that the coefficients adjusted for the local irradiance conditions in Singapore only perform marginally better than the original ones from Perez et al. (1990), confirming the broad applicability of the Perez model for irradiance conditions including the tropics. We note that the model coefficients depend on the datasets; including more data from various tilts may result in a more robust hyperplane fitting. For applications of the Perez model at locations remote from the ones listed in section 1, locally fitted model coefficients may be helpful to improve the conversion accuracies. However, Table 3 suggests that the errors are small enough to be compatible with measurement uncertainties (for example $\pm 3 \%$ for CMP11 from Kipp\&Zonen). 


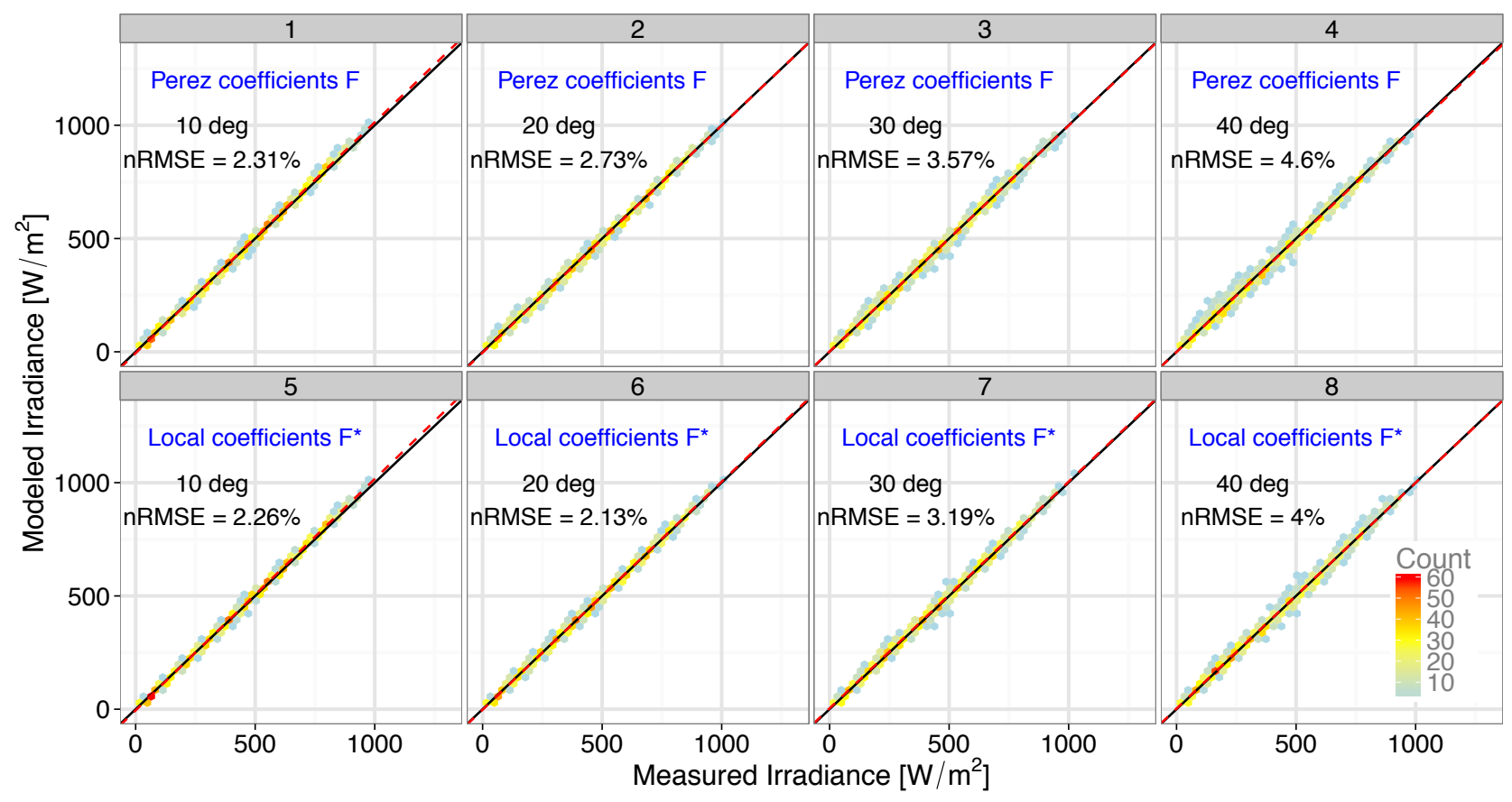

Figure 2: Modeling scatter plots using the coefficients sets $F$ from the original Perez model and $F^{*}$ derived for the tropics. Hexagon binning algorithm is used for visualization. The black solid lines are the identity lines while the red dashed lines are the linearly fitted lines.

\section{Solutions to the inverse problem}

In some instances, it is necessary to apply the inverse Perez model, i.e., converting irradiance from tilt to horizontal. For example, one may seek to leverage in-plane irradiance readings from PV systems (typically made at larger PV systems for performance ratio evaluation) for GHI data, which then can be used to create irradiance maps of an area. With increasing penetration levels of variable solar power into electricity grids, such irradiance maps as well as spatio-temporal solar irradiance forecasting techniques become more and more relevant. One such example where GHI data are required is spacetime kriging (Yang et al., 2013b, 2014). We introduce a novel technique in this section, namely, irradiance conversion from tilt to horizontal using two or more silicon sensors. The merit of this new technique is due to its costeffectiveness, as only silicon sensors (measuring tilted global irradiance) are 
required for this conversion algorithm. Neither tilted diffuse measurements nor pyranometers are needed. The derived data also form an alternative databank to the satellite-derived horizontal irradiance (Ineichen, 2014; Súri and Cebecauer, 2014) which is often limited by the spatial and temporal resolution of the satellite data.

To facilitate our analysis, define $k=I_{h} / D_{h}$, then Eqn. (18) can be rearranged as:

$$
\begin{aligned}
G_{c}= & \frac{k D_{h} \cos \theta(1-y)\left(1-L_{\text {spec }}\right)}{\cos Z}+D_{h} \frac{1+\cos S}{2}\left(1-L_{\text {refl }}\right)\left(1-L_{\text {spec }}\right) \\
& +0.5 \delta D_{h}(1+k)(1-\cos S)\left[1+\sin ^{2}(Z / 2)\right]|\cos (\alpha-\beta)|\left(1-L_{\text {reff }}\right)\left(1-L_{\text {spec }}\right) \\
& +D_{h}\left(\frac{a}{b}-\frac{1+\cos S}{2}\right)\left(1-L_{\text {reff }}\right)\left(1-L_{\text {spec }}\right)\left(F_{11}+\frac{D_{h}}{I_{0} \cos Z} F_{12}+Z F_{13}\right) \\
& +D_{h} \sin S\left(1-L_{\text {refl }}\right)\left(1-L_{\text {spec }}\right)\left(F_{21}+\frac{D_{h}}{I_{0} \cos Z} F_{22}+Z F_{23}\right)
\end{aligned}
$$

We would like to note that the invention of parameter $k$ is strictly for mathematical convenience. One could consider $k$ as a form of $G_{h}$, since $G_{h}=I_{h}+D_{h}$. By inspecting the equation, two cases are made:

- $S=0$, i.e., irradiance readings from horizontal silicon sensors are available. (We note that this is a null problem. Its inclusion is to maintain mathematical completeness.);

- $S>0$, i.e., irradiance readings from horizontal silicon sensors are unavailable.

\subsection{Case of $S=0$}

In this case, $\theta=Z, a=b$, and Eqn. (31) is simplified to

$$
k D_{h}(1-y)\left(1-L_{\text {spec }}\right)+D_{h}\left(1-L_{\text {refl }}\right)\left(1-L_{\text {spec }}\right)=G_{0} .
$$

- If $D_{h}$ is known, $k$ can be solved from Eqn. (32) as

$$
k=\frac{G_{0} / D_{h}-\left(1-L_{\mathrm{refl}}\right)\left(1-L_{\mathrm{spec}}\right)}{(1-y)\left(1-L_{\mathrm{spec}}\right)} .
$$

Thus $I_{h}=k D_{h}$ and $G_{h}=(1+k) D_{h}$. 
- If $k$ is known, from Eqn. (32)

$$
D_{h}=\frac{G_{0}}{k(1-y)\left(1-L_{\mathrm{spec}}\right)+\left(1-L_{\mathrm{reff}}\right)\left(1-L_{\mathrm{spec}}\right)} .
$$

So $I_{h}=k D_{h}$ and $G_{h}=(1+k) D_{h}$.

- If both $D_{h}$ and $k$ are unknown, then with only one equation there exist infinite solutions. There are two ways to deal with the issue. Applying decomposition models to approximate the diffuse irradiance from horizontal irradiance (e.g. Erbs et al., 1982; Maxwell, 1987). However, due to the non-injective nature of the mapping from GHI to DHI, using decomposition models introduces large errors (Ridley et al., 2010). In the other case, irradiance readings from another tilted silicon sensor can assist to find the unique solution by providing the additional equation, which will be discussed in section 3.2 as a special case.

In general, when global irradiance measurements from horizontal silicon sensors $\left(G_{0}\right)$ are available, the derivation of $D_{h}$ and $G_{h}$ becomes straightforward and the Perez model is not required for reconstruction.

\subsection{Case of $S>0$}

In this case, Eqn. (31) can be re-arranged to:

$$
\left(\hat{a} D_{h}+\hat{b} k+\hat{c}\right) D_{h}=G_{c},
$$

where

$$
\begin{aligned}
\hat{a}= & \frac{\left[F_{12}\left(\frac{a}{b}-\frac{1+\cos S}{2}\right)+F_{22} \sin S\right]\left(1-L_{\mathrm{reff}}\right)\left(1-L_{\mathrm{spec}}\right)}{I_{0} \cos Z} \\
\hat{b}= & \frac{\cos \theta(1-y)\left(1-L_{\mathrm{spec}}\right.}{\cos Z}+\left(1-L_{\mathrm{reff}}\right)\left(1-L_{\mathrm{spec}}\right) \\
& \times 0.5 \delta(1-\cos S)\left[1+\sin ^{2}(Z / 2)\right]|\cos (\alpha-\beta)| \\
\hat{c}= & \left(1-L_{\mathrm{reff}}\right)\left(1-L_{\mathrm{spec}}\right)\left\{0.5 \delta(1-\cos S)\left[1+\sin ^{2}(Z / 2)\right]|\cos (\alpha-\beta)|\right. \\
& +\frac{1+\cos S}{2}+\left(F_{11}+Z F_{13}\right)\left(\frac{a}{b}-\frac{1+\cos S}{2}\right) \\
& \left.+\left(F_{21}+Z F_{23}\right) \sin S\right\} .
\end{aligned}
$$


- If $D_{h}$ is known, $k$ can be solved from Eqn. (35) as

$$
k=\frac{G_{c} / D_{h}-\hat{a} D_{h}-\hat{c}}{\hat{b}} .
$$

Thus $I_{h}=k D_{h}$ and $G_{h}=(1+k) D_{h}$.

- If $k$ is known, from Eqn. (35)

$$
\hat{a} D_{h}^{2}+(\hat{b} k+\hat{c}) D_{h}-G_{c}=0 .
$$

If $\hat{a}=0$, the equation has a unique solution:

$$
D_{h}=\frac{G_{c}}{\hat{b} k+\hat{c}} .
$$

If $\hat{a} \neq 0$ and $(\hat{b} k+\hat{c})^{2}+4 \hat{a} G_{c} \geq 0$, Eqn. (35) can be solved as

$$
D_{h}=\frac{-(\hat{b} k+\hat{c}) \pm \sqrt{(\hat{b} k+\hat{c})^{2}+4 \hat{a} G_{c}}}{2 \hat{a}} .
$$

Any negative solution should be ignored as $D_{h} \geq 0$.

- If both $D_{h}$ and $k$ are unknown, as mentioned in section 3.1, irradiance values from another silicon sensor with different tilt are necessary to get the unique solution. Suppose the irradiance values from two different tilted silicon sensors are $G_{c 1}$ and $G_{c 2}$, respectively, it follows from Eqn. (35) that

$$
\begin{aligned}
\left(a_{1} D_{h}+b_{1} k+c_{1}\right) D_{h} & =G_{c 1}, \\
\left(a_{2} D_{h}+b_{2} k+c_{2}\right) D_{h} & =G_{c 2} .
\end{aligned}
$$

Mutually dividing Eqns. (43) and (44) to eliminate $D_{h}$ yields

$$
k=\frac{\left(a_{2} G_{c 1}-a_{1} G_{c 2}\right) D_{h}+c_{2} G_{c 1}-c_{1} G_{c 2}}{b_{1} G_{c 2}-b_{2} G_{c 1}} .
$$

Substituting Eqn. (45) into Eqn. (43) yields

$$
a^{*} D_{h}^{2}+b^{*} D_{h}+c^{*}=0,
$$


where

$$
\begin{aligned}
a^{*} & =a_{1}+b_{1} \frac{a_{2} G_{c 1}-a_{1} G_{c 2}}{b_{1} G_{c 2}-b_{2} G_{c 1}}, \\
b^{*} & =c_{1}+b_{1} \frac{c_{2} G_{c 1}-c_{1} G_{c 2}}{b_{1} G_{c 2}-b_{2} G_{c 1}}, \\
c^{*} & =-G_{c 1} .
\end{aligned}
$$

If $a^{*}=0$, the group of Eqns. (43) and (44) has a unique solution:

$$
D_{h}=-\frac{c^{*}}{b^{*}}
$$

If $a^{*} \neq 0$ and $b^{* 2}-4 a^{*} c^{*} \geq 0$, the two solutions to Eqns. (43) and (44) are

$$
D_{h}=\frac{-b^{*} \pm \sqrt{b^{* 2}-4 a^{*} c^{*}}}{2 a^{*}},
$$

where the negative root should be ignored.

The closed form solutions presented above cover all cases of data availability. We select model coefficients $F_{11}, F_{12}, F_{13}, F_{21}, F_{22}, F_{23}$ in the following manner:

- Values of defined coefficients $\hat{a}, \hat{b}$ and $\hat{c}$ are only functions of $F_{11}, F_{12}$, $F_{13}, F_{21}, F_{22}, F_{23}$.

- Eight sets of $\hat{a}, \hat{b}$ and $\hat{c}$ can be calculated based on different Perez model coefficients. These values lead to eight diffuse horizontal irradiance (DHI) estimates, thus eight global horizontal irradiance (GHI) estimates.

- Eight $\varepsilon$ values can be then calculated using GHI/DHI estimates.

- If the assumed $\varepsilon$ agrees with the calculated $\varepsilon$, the set is selected as the true estimated GHI. Furthermore, direct normal irradiance (DNI) can be calculated using true estimates of GHI.

\subsection{Least square solution}

In the previous sections, only unique solutions are discussed. If the irradiance readings from multiple silicon sensors (with different inclinations) 
are known, the least square solution should be available. Let $X=\left(D_{h}, k\right)^{\prime}$, $A=\left(\begin{array}{cc}a & b / 2 \\ b / 2 & 0\end{array}\right), B=(c, 0), C=G_{c}$, Eqn. (35) in matrix format is given by

$$
X^{\prime} A X+B X-C=0
$$

When multiple $G_{c}$ with different inclinations are available, Eqn. (52) becomes

$$
X^{\prime} \Lambda X+\mathbf{B} X-\mathbf{C}=0
$$

where $\boldsymbol{\Lambda}=\left\{A_{i}\right\} \in \mathbb{R}^{2 \times m \times 2}$ is a three-order tensor, $\mathbf{B}=\left\{B_{i}\right\} \in \mathbb{R}^{m \times 2}$, $\mathbf{C}=\left\{C_{i}\right\} \in \mathbb{R}^{m}, i=1,2, \cdots, m$, with $m>2$. Thus, the least square solution is to minimize

$$
\min \left\{P(X)=\frac{1}{2}\left\|X^{\prime} \boldsymbol{\Lambda} X+\mathbf{B} X-\mathbf{C}=0\right\|^{2}: X \in \mathbb{R}^{2}\right\},
$$

where $\|\cdot\|$ denotes the Euclidean norm.

In general, the target function $P(X)$ is a fourth-order polynomial, which may have multiple local extremum solutions. The standard techniques for solving non-convex problems are mainly Newton type iteration methods (Grippo and Lucidi, 1991; Grosan and Abraham, 2008). It was shown in (Gao and Ogden, 2008) that the solutions to non-convex minimization problems are usually non-smooth and cannot be captured by any kind of Newton type direct approaches. Due to the lack of global optimality conditions, many non-convex minimization problems in global optimization are considered as "NP-hard", i.e., "nondeterministic polynomial time hard".

Ruan et al. (2010) proved that, by applying the canonical duality theory, the above non-convex problem is equivalent to a concave maximization problem in $\mathbb{R}^{m}$, which can be solved easily by well-developed convex optimization techniques. However, this technique goes beyond the field of solar energy and the unique solution presented in section 3.1 and 3.2 is accurate enough for our analysis, especially when taking into account the uncertainties in the measurements.

\subsection{Case studies}

With the reliable data of 5 months (2014 January to May), the inverse problem of Perez transposition is validated using 10 case studies:

(1) $D_{h}$ is known, reconstruct $G_{h}$ from $G_{10}\left(10^{\circ}\right.$ tilted silicon sensor); 
(2) $D_{h}$ is known, reconstruct $G_{h}$ from $G_{20}$ (20 tilted silicon sensor);

(3) $D_{h}$ is known, reconstruct $G_{h}$ from $G_{30}$ (30 tilted silicon sensor);

(4) $D_{h}$ is known, reconstruct $G_{h}$ from $G_{40}$ ( $40^{\circ}$ tilted silicon sensor);

(5) $D_{h}$ is unknown, reconstruct $G_{h}$ from $G_{10}$ and $G_{20}$;

(6) $D_{h}$ is unknown, reconstruct $G_{h}$ from $G_{10}$ and $G_{30}$;

(7) $D_{h}$ is unknown, reconstruct $G_{h}$ from $G_{10}$ and $G_{40}$;

(8) $D_{h}$ is unknown, reconstruct $G_{h}$ from $G_{20}$ and $G_{30}$;

(9) $D_{h}$ is unknown, reconstruct $G_{h}$ from $G_{20}$ and $G_{40}$;

(10) $D_{h}$ is unknown, reconstruct $G_{h}$ from $G_{30}$ and $G_{40}$.

These 10 case studies cover all sensor permutations. In addition to these, we also define another 8 case studies whose choices are justified in section 3.5.

(11) $D_{h}$ is unknown, reconstruct $G_{h}$ from $G_{10}, G_{20}$ and $G_{30}$;

(12) $D_{h}$ is unknown, reconstruct $G_{h}$ from $G_{10}, G_{30}$ and $G_{40}$;

(13) $D_{h}$ is unknown, reconstruct $G_{h}$ from $G_{20}, G_{30}$ and $G_{40}$;

(14) $D_{h}$ is unknown, reconstruct $G_{h}$ from $G_{10}, G_{20}, G_{30}$ and $G_{40}$;

(15) $D_{h}$ is unknown, reconstruct $G_{h}$ from $G_{10}$ only (with decomposition mode);

(16) $D_{h}$ is unknown, reconstruct $G_{h}$ from $G_{20}$ only (with decomposition mode);

(17) $D_{h}$ is unknown, reconstruct $G_{h}$ from $G_{30}$ only (with decomposition mode);

(18) $D_{h}$ is unknown, reconstruct $G_{h}$ from $G_{40}$ only (with decomposition mode).

Table 4 shows the error metrics for reconstructions, which follows Eqns. (28), (29) and (30) with $G_{h}$ instead of $G_{c}$. The errors are observed to be in the same range as those reported in Table 3 . We also note that by using two silicon sensors on different tilts (case studies (5), (6) and (7)), it is possible to produce better results than deriving the $D_{h}$ from data of the SPN1 sensor (case studies (3) and (4)). Therefore a pair of carefully modeled silicon sensors is preferred over a suboptimal grade pyranometer due to the lower cost of silicon sensors.

\subsection{Benchmarking}

The studies on inverse transposition is rarely encountered in the literature. Nevertheless, the works by Faiman et al. $(1993,1987)$ reveal a simplistic approach to the inverse transposition problem. In both works, a multipyranometer approach is used to solve the problem by first assuming isotropy in both diffuse irradiance and irradiance due to ground reflection. We implement the method outlined by Faiman et al. (1987) to benchmark our results. 
Table 4: Errors from the proposed method to solve the inverse Perez model. Faiman et al. (1987) model and Yang et al. (2013a) model are used to benchmark the results. The mean observed global horizontal irradiance is $470.06 \mathrm{~W} / \mathrm{m}^{2}$.

\begin{tabular}{|c|c|c|c|c|c|c|c|c|c|}
\hline \multirow[b]{2}{*}{ Case } & \multicolumn{3}{|c|}{ Inverse Perez model } & \multicolumn{3}{|c|}{ Faiman et al. (1987) model } & \multicolumn{3}{|c|}{ Yang et al. (2013a) model } \\
\hline & $\begin{array}{c}\mathrm{MBE} \\
{\left[\mathrm{W} / \mathrm{m}^{2}\right]}\end{array}$ & $\begin{array}{c}\text { RMSE } \\
{[\%]}\end{array}$ & $\begin{array}{l}U_{95} \\
{[\%]}\end{array}$ & $\begin{array}{c}\mathrm{MBE} \\
{\left[\mathrm{W} / \mathrm{m}^{2}\right]}\end{array}$ & $\begin{array}{c}\text { RMSE } \\
{[\%]}\end{array}$ & $\begin{array}{l}U_{95} \\
{[\%]}\end{array}$ & $\begin{array}{c}\mathrm{MBE} \\
{\left[\mathrm{W} / \mathrm{m}^{2}\right]}\end{array}$ & $\begin{array}{c}\text { RMSE } \\
{[\%]}\end{array}$ & $\begin{array}{l}U_{95} \\
{[\%]}\end{array}$ \\
\hline (1) & -4.12 & 1.87 & 3.28 & & - & & & - & \\
\hline (2) & -0.02 & 1.69 & 3.32 & & - & & & - & \\
\hline (3) & -3.26 & 2.64 & 5.02 & & - & & & - & \\
\hline (4) & 0.55 & 3.84 & 7.53 & & - & & & - & \\
\hline (5) & -3.88 & 2.57 & 4.58 & -7.68 & 5.11 & 9.47 & & - & \\
\hline (6) & -4.53 & 2.43 & 4.30 & -5.61 & 4.63 & 8.76 & & - & \\
\hline (7) & -3.86 & 2.33 & 4.23 & -6.42 & 5.01 & 9.44 & & - & \\
\hline (8) & 1.30 & 3.21 & 6.27 & 1.38 & 5.45 & 10.67 & & - & \\
\hline (9) & 0.73 & 2.70 & 5.28 & -0.18 & 5.04 & 9.87 & & - & \\
\hline (10) & 3.06 & 5.66 & 11.01 & -6.54 & 7.32 & 14.08 & & - & \\
\hline (11) & & - & & -5.87 & 3.61 & 6.63 & & - & \\
\hline (12) & & - & & -6.16 & 3.71 & 6.80 & & - & \\
\hline (13) & & - & & -0.64 & 4.08 & 7.99 & & - & \\
\hline (14) & & - & & -5.01 & 3.49 & 6.50 & & - & \\
\hline$(15)$ & & - & & & - & & 1.11 & 4.01 & 7.85 \\
\hline (16) & & - & & & - & & 3.75 & 7.27 & 14.17 \\
\hline (17) & & - & & & - & & -13.68 & 16.13 & 31.09 \\
\hline (18) & & - & & & - & & -26.72 & 23.00 & 43.65 \\
\hline
\end{tabular}

With isotropic assumptions on the diffuse transposition factor and the transposition factor due to ground reflection, Eqn. (14) is simplified to:

$$
\begin{aligned}
D_{c}= & 0.5 \delta\left(I_{h}+D_{h}\right)(1-\cos S)\left(1-L_{\mathrm{refl}}\right)\left(1-L_{\mathrm{spec}}\right) \\
& +D_{h} \frac{1+\cos S}{2}\left(1-L_{\mathrm{refl}}\right)\left(1-L_{\mathrm{spec}}\right)
\end{aligned}
$$

Together with the in-plane direct irradiance, we substitute Eqns. (55) and (16) into $G_{c}=I_{c}+D_{c}$ and yield:

$$
\begin{aligned}
G_{c}= & 0.5 \delta\left(I_{h}+D_{h}\right)(1-\cos S)\left(1-L_{\mathrm{reff}}\right)\left(1-L_{\mathrm{spec}}\right) \\
& +D_{h} \frac{1+\cos S}{2}\left(1-L_{\mathrm{refl}}\right)\left(1-L_{\mathrm{spec}}\right) \\
& +I_{h} \frac{\cos \theta}{\cos Z}(1-y)\left(1-L_{\mathrm{spec}}\right) .
\end{aligned}
$$

By inspecting, Eqn. (56) can be conveniently written into:

$$
G_{c}=p I_{h}+q D_{h}
$$


with

$$
\begin{aligned}
& p=\left[\frac{\cos \theta}{\cos Z}(1-y)+\delta\left(\frac{1-\cos S}{2}\right)\left(1-L_{\mathrm{refl}}\right)\right]\left(1-L_{\mathrm{spec}}\right) \\
& q=\left[\frac{(1+\cos S)+\delta(1-\cos S)}{2}\right]\left(1-L_{\mathrm{refl}}\right)\left(1-L_{\mathrm{spec}}\right)
\end{aligned}
$$

which can be readily calculated. Given $n$ tilted sensors, this equation can then be written into linear algebra form:

$$
\tilde{\mathbf{A}} \tilde{\mathbf{X}}=\tilde{\mathbf{Y}}
$$

where

$$
\begin{aligned}
& \tilde{\mathbf{X}}=\left(\begin{array}{ll}
I_{h} & D_{h}
\end{array}\right)^{\prime}, \quad \in \mathbb{R}^{2} \\
& \tilde{\mathbf{Y}}=\left(\begin{array}{lll}
G_{c}^{1} & \cdots & G_{c}^{n}
\end{array}\right)^{\prime}, \quad \in \mathbb{R}^{n}
\end{aligned}
$$

and

$$
\tilde{\mathbf{A}}=\left(\begin{array}{cc}
p_{1} & q_{1} \\
\vdots & \vdots \\
p_{n} & q_{n}
\end{array}\right) . \quad \in \mathbb{R}^{n \times 2}
$$

The least square solution to Eqn. (60) is given by:

$$
\tilde{\mathbf{X}}=\left(\tilde{\mathbf{A}}^{\prime} \tilde{\mathbf{A}}\right)^{-1} \tilde{\mathbf{A}}^{\prime} \tilde{\mathbf{Y}}
$$

Based on Eqn. (64), $I_{h}$ and $D_{h}$ can be calculated based on data from any $n \geq 2$ sensors, and thus $G_{h}$. We use the Faiman et al. (1987) model in case studies (5) to (14) covering all sensors permutations. Table 4 shows the error metrics of the model. It can be seen that the isotropic Faiman et al. (1987) model only performs marginally worse than the proposed inverse Perez model. Further to that, when 3 or more sensors are used (case studies (11) to (14)), the errors are smaller than the errors from the 2 sensors cases of the Faiman et al. (1987) model.

These above comparison between the isotropic and anisotropic approaches to the inverse transposition problem aligns with the results reported in a previous work by Gueymard (2009) which evaluates the horizontal to tilt conversions. The improvements from using isotropic models to using anisotropic models in both works are shown to be certain but marginal. We note that the Perez model depends on $\varepsilon$ binning, the retrieved $\varepsilon$ values might not agree 
with the expected bin. Thus, when we follow the procedures described in section 3.2, the conversion of a small percentage of data points will be unsuccessful. The failure rate of the algorithm is about 2 to 3 percent (see the supplementary materials for details). This issue however can be addressed by redefining the $\varepsilon$ binning. On the other hand, the isotropic approach has guaranteed solutions. The trade-off between the isotropic and anisotropic approaches can be evaluated based on the applications.

All of the tilt to horizontal conversion algorithms shown so far use at least two sensors in GHI reconstruction. However, more than common, there is only a single sensor available. In this case, the tilt to horizontal conversion can still be carried out. In an earlier work by Yang et al. (2013a), a single tilted silicon sensor is used to reconstruct GHI through a combination of transposition and decomposition models. Decomposition models, which can predict $D_{h}$ using $G_{h}$, are used owing to the fact that at least two sets of irradiance measurements are needed for GHI reconstruction (see case studies (1) to (10) above). As a tilted silicon sensor can provide one set of irradiance measurements, the other set of measurements can be obtained through the decomposition models.

Erbs univariate decomposition model (Erbs et al., 1982) was considered by Yang et al. (2013a). Irradiance data collected using silicon sensors on two tilted planes, namely, a $18.3^{\circ} \mathrm{NE}$ facing plane and a $6.1^{\circ} \mathrm{SW}$ facing plane, were individually used to reconstruct the GHI measurements made using a SPN1 pyranometer. RMSE of $\approx 13 \%$ and $\approx 6 \%$ were found for the two tilts respectively (see Yang et al., 2013a, for details). We apply these techniques using our current setup and data in case studies (15) to (18), the tilt to horizontal conversion errors using a single tilted silicon sensor are shown in Table 4.

The RMSE calculated using measurements from the $10^{\circ}$ tilt and the $20^{\circ}$ tilt sensors improve from the previous study as various losses are accounted in this paper. Since the mapping between GHI and DHI is non-injective owing to different sky conditions, the errors using the Yang et al. (2013a) model are much larger than the ones using multiple sensor approaches. We thus conclude that by including another reference cell at an alternative tilt, the accuracy of tilt to horizontal conversion can be improved substantially. We have shown that the uncertainties of the proposed algorithm are within the uncertainties of the measurements. The proposed conversion algorithm is therefore well suited and sophisticated enough to describe the experimental results; a further refinement is not necessary. 


\section{Conclusion}

A least squares method is first introduced to optimize the Perez transposition model using the tropical data. The locally fitted set of coefficients outperforms the original set of coefficients. We promote such regional optimizations of the Perez model coefficients when data are available.

This paper establishes a method for two-way conversion among various irradiance components received on a horizontal plane and the ones received on tilted planes using the Perez model. Three irradiance components are defined on a horizontal plane, namely, $G_{h}, D_{h}$ and $I$. On an arbitrary tilted plane, $G_{c 1}$ and $D_{c 1}$ can be defined; similarly, $G_{c 2}$ and $D_{c 2}$ can be defined at an alternative tilt. Following the presented methodologies, when 2 out of 7 components are known, we can model all other components deterministically. This algorithm thus gives convenience to a series of solar engineering problems, including:

- Use $G_{h}$ (pyranometer) and $D_{h}$ (another pyranometer with shadow band or shadow pattern) measurements to model $G_{c}$ for PV module performance benchmarking. The second pyranometer can be replaced by a pyrheliometer who measures $I$.

- Use measured $G_{c 1}$ and $G_{c 2}$ (two reference cells) to reconstruct $G_{h}$ and $D_{h}$. The reconstructions are used to generate GHI maps and as inputs to irradiance forecasting algorithms (e.g. Yang et al., 2013b,c; Lonij et al., 2013). This can be enhanced with tilted pyranometers, with equipment cost being the trade-off.

- Use $G_{h}$ (pyranometer) and $G_{c}$ (reference cell) to reconstruct the often desired horizontal component, $D_{h}$, for financial saving on equipments.

Of particular importance to the methods introduced in this paper is that observations need not use costly pyranometers, but can be derived from lowcost reference cells. Moreover, we show how measurements taken in the plane of a tilted PV system can be used. Since such observations are very commonly carried out in larger PV systems, we greatly increase the potential number of sites that can benefit from a locally-optimized Perez model, even in the absence of a research-grade meteorological station. We apply our method to the tropical location of Singapore: the derived results find that a reasonable fit is in fact provided by the original (Perez et al., 1990) coefficients, but we note that there is no reason to suppose that this will be the case at 
all locations. Both of the techniques introduced in this paper will become increasingly relevant for the optimal management of variable PV output into electricity power grids around the world.

\section{Supplementary materials}

This paper comes with supplementary materials. We provide the $\mathrm{R}$ code used to generate the results in section 3. Instead of providing the original Singapore dataset, we use typical meteorological year (TMY3) files in the code to protect proprietary information. The TMY3 data are freely online at http://rredc.nrel.gov/solar/old_data/nsrdb/1991-2005/tmy3/. The user manual of the datasets is found at the same website.

In the code provided, we choose a class I site, Los Angeles international airport with USAFN number 722950. GHI and DHI values from the files are used to simulate two sets of hourly tilted irradiance using the Perez model. The tilt angles and the azimuth angles can be set arbitrarily by users. These generated tilted irradiance values are then used to reconstruct the GHI and DHI values. The reconstructed GHI values show negligible discrepancy (RMSE $\ll 1 \%$ when $D_{h}$ is known and $<2 \%$ when $D_{h}$ is unknown) as compared to the measured GHI, proving the deterministic nature of the proposed inverse transposition model. We conclude that the inverse transposition model's accuracy is only limited by the accuracy of the Perez model and data measurement uncertainty.

\section{Appendix A. Reflection loss models}

In section 2 of the paper, we account for the reflection loss of $I_{c}$ on the glass surface of reference cells using an empirical piecewise linear function

of incidence angle fitted by the Fraunhofer ISE, see Eqn. (17). We clarify certain model assumptions here.

Reflection loss in PV is commonly modeled as functions of the incidence angle (e.g. Martín and Ruiz, 2005). Martin and Ruiz (2001) proposed an analytical model which has been validated using experimental data:

$$
y=1-\left[\frac{1-\exp \left(-\frac{\cos \theta}{a_{r}}\right)}{1-\exp \left(-\frac{1}{a_{r}}\right)}\right]
$$


where $\theta$ is the incidence angle and $a_{r}$ is the angular loss coefficient. Model parameter $a_{r}$ depends on the PV configuration. For examples, $a_{r}=0.136$ corresponds to a "air-glass-a-Si:H-Ag" configuration (Martin and Ruiz, 2001) and $a_{r}=0.2$ corresponds to a "air-glass" configuration with a moderately soiled surface (Martín and Ruiz, 2005). These two example configurations are shown as the lower and upper bounds in Figure A.3 (a) respectively. We refer the model in Eqn. (A.1) as the M\&R model hereafter. The gray band in Figure A.3 (a) shows the M\&R model with $0.2 \geq a_{r} \geq 0.136$. In Figure A.3 (b), we benchmark the Fraunhofer model with the $\operatorname{M} \& \mathrm{R}\left(a_{r}=0.173\right)$ model, which corresponds to a "air-glass" configuration without soiling (Martin and Ruiz, 2001). Our sensors are cleaned weekly by our co-workers, $a_{r}=0.173$ is therefore a suitable choice.

The M\&R model is a continuous function, which provides a smooth mapping between the incidence angle and the reflection loss. The Fraunhofer model is not differentiable everywhere. Although the M\&R model gives a more comprehensive representation of the reflection loss in PV, the angular loss coefficient needs to be determined based on particular devices. On the other hand, the Fraunhofer model is developed and fitted using the same instruments as the ones herein used. We consider the Fraunhofer model to be more suitable in our applications. In other circumstances, to use a M\&R model, it is convenient to replace reflection loss $y$ in Eqn. (17) with the one in Eqn. (A.1). The other steps in the algorithms presented in this paper remain unchanged.

Erbs, D.G., Klein, S.A., Duffie, J.A., 1982. Estimation of the diffuse radiation fraction for hourly, daily and monthly-average global radiation. Solar Energy 28, $293-302$.

Faiman, D., Feuermann, D., Zemel, A., 1993. Site-independent algorithm for obtaining the direct beam insolation from a multipyranometer instrument. Solar Energy 50, 53 - 57. URL: http://www. sciencedirect.com/ science/article/pii/0038092X9390007B, doi:10.1016/0038-092X (93) 90007-B.

Faiman, D., Zemel, A., Zangvil, A., 1987. A method for monitoring insolation in remote regions. Solar Energy 38, 327 - 333. URL: http: //www.sciencedirect.com/science/article/pii/0038092X87900041, doi:http://dx.doi.org/10.1016/0038-092X (87)90004-1. 

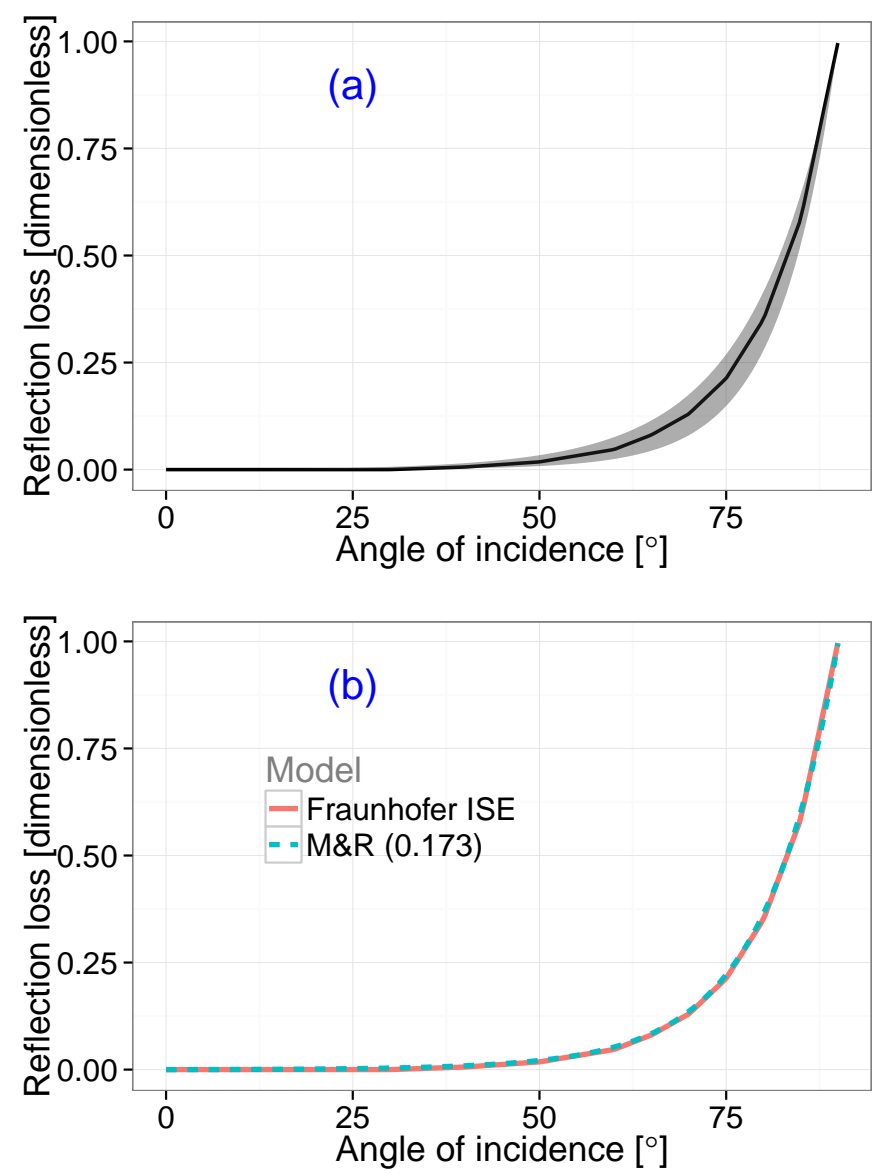

Figure A.3: Comparison of reflection loss models. Top plot (a) shows the Fraunhofer model (solid line) is well within the M\&R model bounds (gray region). Bottom plot (b) shows the precise agreement between the Fraunhofer model and the M\&R model with a "air/glass" configuration, i.e., the $a_{r}=0.173$ case. 
Gao, D.Y., Ogden, R.W., 2008. Multiple solutions to non-convex variational problems with implications for phase transitions and numerical computation. The Quarterly Journal of Mechanics and Applied Mathematics 61, 497-522. URL: http://qjmam . oxfordjournals .org/content/61/4/ 497. abstract, doi:10.1093/qjmam/hbn014.

Grippo, L., Lucidi, S., 1991. A differentiable exact penalty function for bound constrained quadratic programming problems. Optimization 22, $557-578$.

Grosan, C., Abraham, A., 2008. A new approach for solving nonlinear equations systems. Systems, Man and Cybernetics, Part A: Systems and Humans, IEEE Transactions on 38, 698-714. doi:10.1109/TSMCA.2008. 918599.

Gueymard, C.A., 2009. Direct and indirect uncertainties in the prediction of tilted irradiance for solar engineering applications. Solar Energy 83, 432 - 444. URL: http://www. sciencedirect.com/science/article/ pii/S0038092X08002983, doi:http://dx.doi.org/10.1016/j.solener. 2008.11.004.

Hay, J.E., 1979. Calculation of monthly mean solar radiation for horizontal and inclined surfaces. Solar Energy 23, 301 - 307. URL: http: //www.sciencedirect.com/science/article/pii/0038092X79901233, doi:http://dx.doi.org/10.1016/0038-092X (79) 90123-3.

Ineichen, P., 2014. Long term satellite global, beam and diffuse irradiance validation. Energy Procedia 48, 1586 - 1596. URL: http:// www.sciencedirect.com/science/article/pii/S187661021400441X, doi:http://dx.doi.org/10.1016/j.egypro.2014.02.179. proceedings of the 2nd International Conference on Solar Heating and Cooling for Buildings and Industry (SHC 2013).

Khoo, Y.S., Nobre, A., Malhotra, R., Yang, D., Ruther, R., Reindl, T., Aberle, A., 2014. Optimal orientation and tilt angle for maximizing inplane solar irradiation for PV applications in Singapore. Photovoltaics, IEEE Journal of 4, 647-653. doi:10.1109/JPHOTOV . 2013.2292743.

Klucher, T., 1979. Evaluation of models to predict insolation on tilted surfaces. Solar Energy 23, 111 - 114. URL: http: 
//www.sciencedirect.com/science/article/pii/0038092X79901105, doi:http://dx.doi.org/10.1016/0038-092X (79) 90110-5.

Liu, B.Y.H., Jordan, R.C., 1962. Daily insolation on surfaces tilted towards the equator. Transactions ASHRAE 67, 526-541.

Lonij, V.P., Brooks, A.E., Cronin, A.D., Leuthold, M., Koch, K., 2013. Intra-hour forecasts of solar power production using measurements from a network of irradiance sensors. Solar Energy 97, 58 - 66. URL: http://www.sciencedirect.com/science/article/ pii/S0038092X13003125, doi:http://dx.doi.org/10.1016/j.solener. 2013.08.002.

Ma, C., Iqbal, M., 1983. Statistical comparison of models for estimating solar radiation on inclined surgaces. Solar Energy 31, 313 - 317. URL: http: //www.sciencedirect.com/science/article/pii/0038092X83900191, doi:http://dx.doi.org/10.1016/0038-092X (83)90019-1.

Martin, N., Ruiz, J., 2001. Calculation of the PV modules angular losses under field conditions by means of an analytical model. Solar Energy Materials and Solar Cells 70, 25 38. URL: http://www.sciencedirect.com/science/article/pii/ S0927024800004086, doi:http://dx.doi.org/10.1016/S0927-0248(00) 00408-6.

Martín, N., Ruiz, J.M., 2005. Annual angular reflection losses in PV modules. Progress in Photovoltaics: Research and Applications 13, 75-84. URL: http://dx.doi.org/10.1002/pip.585, doi:10.1002/pip. 585.

Masters, G.M., 2004. Renewable and Efficient Electric Power Systems. John Wiley \& Sons, Inc., Hoboken-New Jersey.

Matagne, E., Bachtiri, R.E., 2014. Exact analytical expression of the hemispherical irradiance on a sloped plane from the perez sky. Solar Energy 99, 267 - 271. URL: http://www.sciencedirect.com/science/article/ pii/S0038092X13004969, doi:http://dx.doi.org/10.1016/j.solener . 2013.11.016.

Maxwell, E.L., 1987. Quasi-physical model for converting hourly global horizontal to direct normal insolation. <http://www.nrel.gov/docs/ leg-osti/old/3087.pdf>. (accessed 25.01.2012). 
Meydbray, J., Emery, K., Kurtz, S., 2012. Pyranometers and reference cells, what's the difference? Technical Report JA-5200-54498. National Renewable Energy Laboratory. Golden, CO.

Michalsky, J.J., 1988. The astronomical almanac's algorithm for approximate solar position (1950 - 2050). Solar Energy 40, 227 235. URL: http://www.sciencedirect.com/science/article/pii/ 0038092X8890045X, doi:10.1016/0038-092X (88) 90045-X.

Myers, D.R., 2012. 1.12 - solar radiation resource assessment for renewable energy conversion, in: Sayigh, A. (Ed.), Comprehensive Renewable Energy. Elsevier, Oxford, pp. 213-237. URL: http://www. sciencedirect.com/ science/article/pii/B9780080878720001128.

Myers, D.R., 2013. Solar Radiation: Practical Modeling for Renewable Energy Applications. CRC Press.

Perez, R., Ineichen, P., Seals, R., Michalsky, J., Stewart, R., 1990. Modeling daylight availability and irradiance components from direct and global irradiance. Solar Energy 44, 271 - 289. URL: http://www. sciencedirect.com/science/article/pii/0038092X9090055H, doi:10. 1016/0038-092X (90) 90055-H.

Perez, R., Seals, R., Ineichen, P., Stewart, R., Menicucci, D., 1987. A new simplified version of the perez diffuse irradiance model for tilted surfaces. Solar Energy 39, 221 231. URL: http://www.sciencedirect.com/science/article/pii/ S0038092X87800312, doi:10.1016/S0038-092X (87) 80031-2.

Perez, R., Stewart, R., Arbogast, C., Seals, R., Scott, J., 1986. An anisotropic hourly diffuse radiation model for sloping surfaces: Description, performance validation, site dependency evaluation. Solar Energy 36, 481 - 497. URL: http://www.sciencedirect.com/science/article/pii/ 0038092X86900137, doi:10.1016/0038-092X (86) 90013-7.

Perez, R., Stewart, R., Seals, R., Guertin, T., 1988. The Development and Verification of the Perez Diffuse Radiation Model. Technical Report SAND88- 7030. Atmospheric Sciences Research Center, SUNY at Albany. Albany, NY. 
Reda, I., Andreas, A., 2004. Solar position algorithm for solar radiation applications. Solar Energy 76, 577 - 589. URL: http:// wWw.sciencedirect.com/science/article/pii/S0038092X0300450X, doi:http://dx.doi.org/10.1016/j.solener.2003.12.003.

Reda, I., Andreas, A., 2008. Solar Position Algorithm for Solar Radiation Applications. Technical Report TP-560-34302. National Renewable Energy Laboratory. Golden, CO.

Ridley, B., Boland, J., Lauret, P., 2010. Modelling of diffuse solar fraction with multiple predictors. Renewable Energy 35, 478 483. URL: http://www.sciencedirect.com/science/article/pii/ S0960148109003012, doi:10.1016/j.renene.2009.07.018.

Ruan, N., Gao, D.Y., Jiao, Y., 2010. Canonical dual least square method for solving general nonlinear systems of quadratic equations. Comput. Optim. Appl. 47, 335-347. URL: http://dx.doi.org/10.1007/ s10589-008-9222-5, doi:10.1007/s10589-008-9222-5.

Skartveit, A., Olseth, J.A., 1986. Modelling slope irradiance at high latitudes. Solar Energy 36, 333 - 344. URL: http://www.sciencedirect.com/ science/article/pii/0038092X86901519, doi:http://dx.doi.org/10. 1016/0038-092X (86) 90151-9.

Temps, R.C., Coulson, K., 1977. Solar radiation incident upon slopes of different orientations. Solar Energy 19, 179 - 184. URL: http: //www.sciencedirect.com/science/article/pii/0038092X77900561, doi:http://dx.doi .org/10.1016/0038-092X (77)90056-1.

Šúri, M., Cebecauer, T., 2014. Satellite-based solar resource data: model validation statistics versus user's uncertainty, in: ASES SOLAR 2014 Conference, San Francisco.

Yang, D., Dong, Z., Nobre, A., Khoo, Y.S., Jirutitijaroen, P., Walsh, W.M., 2013a. Evaluation of transposition and decomposition models for converting global solar irradiance from tilted surface to horizontal in tropical regions. Solar Energy 97, 369 - 387. URL: http://www.sciencedirect.com/science/article/ pii/S0038092X13003435, doi:http://dx.doi.org/10.1016/j.solener. 2013.08 .033$. 
Yang, D., Dong, Z., Reindl, T., Jirutitijaroen, P., Walsh, W.M., 2014. Solar irradiance forecasting using spatio-temporal empirical kriging and vector autoregressive models with parameter shrinkage. Solar Energy 103, 550 - 562. URL: http://www. sciencedirect.com/science/article/ pii/S0038092X14000425, doi:http://dx.doi.org/10.1016/j.solener. 2014.01 .024 .

Yang, D., Gu, C., Dong, Z., Jirutitijaroen, P., Chen, N., Walsh, W.M., 2013b. Solar irradiance forecasting using spatial-temporal covariance structures and time-forward kriging. Renewable Energy 60, 235 245. URL: http://www.sciencedirect.com/science/article/pii/ S0960148113002759, doi:10.1016/j.renene.2013.05.030.

Yang, D., Walsh, W.M., Zibo, D., Jirutitijaroen, P., Reindl, T.G., 2013c. Block matching algorithms: Their applications and limitations in solar irradiance forecasting. Energy Procedia 33, 335 342. URL: http://www.sciencedirect.com/science/article/pii/ S1876610213000842, doi:http://dx.doi.org/10.1016/j. egypro. 2013. 05.074. pV Asia Pacific Conference 2012.

Zomer, C., Nobre, A., Cassatella, P., Reindl, T., Ruther, R., 2013. The balance between aesthetics and performance in building-integrated photovoltaics in the tropics. Progress in Photovoltaics: Research and Applications URL: http://dx.doi.org/10.1002/pip.2430, doi:10.1002/pip. 2430 . 